\title{
Shear stress induces human aortic endothelial cell apoptosis via interleukin-1 receptor-associated kinase 2-induced endoplasmic reticulum stress
}

\author{
LONGFEI PAN ${ }^{1}$, ZHOU HONG $^{2}$, LEI YU ${ }^{3}$, YANXIA GAO ${ }^{1}$, \\ RUI ZHANG ${ }^{1}$, HUI FENG ${ }^{1}$, LIJUAN SU ${ }^{1}$ and GANG WANG ${ }^{1}$ \\ ${ }^{1}$ Department of Emergency Medicine, The Second Affiliated Hospital of Xi'an Jiaotong University, Xi'an, \\ Shaanxi 710004; ${ }^{2}$ Department of Emergency Medicine, The Fifth Hospital of Xi'an, Xi'an, Shaanxi 710082; \\ ${ }^{3}$ Department of Basic Medicine, Xi'an Medical College, Xi'an, Shaanxi 710068, P.R. China
}

Received August 15, 2016; Accepted May 12, 2017

DOI: $10.3892 / \mathrm{mmr} .2017 .7524$

\begin{abstract}
Atherosclerosis is characterized by localized lesions distributed in the arterial tree due to the shear stress produced by blood flow. Endothelial cells are directly affected by alterations in blood flow. Dysfunction and injury to endothelial cells has been hypothesized to initiate the pathological processes of atherosclerosis. The present study aimed to investigate the mechanism of shear stress-induced endothelial cellular apoptosis. Shear stress was generated using an artificial device to mimic the impact of disturbed blood flow on cultured human aortic endothelial cells (HAECs). Cellular apoptosis was assessed using a terminal deoxynucleotidyl transferase dUTP nick end labeling assay; an ELISA assay was used to detect the produced interleukin (IL)-1 $\beta$; specific small interfering (si)RNA was used to knockdown the expression of interleukin-1 receptor-associated kinase 2 (IRAK2) in HAECs and the expression levels of $78 \mathrm{kDa}$ glucose-regulated protein, DNA damage-inducible transcript 3 protein (CHOP), IRAK2 and IL-1 $\beta$ were evaluated using western blotting. The results of the present study demonstrated that artificial shear stress induced endoplasmic reticulum (ER) stress, IL- $1 \beta$ production and apoptosis in HAECs in a time-dependent manner. The inhibition of ER stress, and treatment with interleukin-1 receptor antagonist protein and siRNA against IRAK2 attenuated shear stress-induced CHOP signaling-mediated cellular apoptosis. Therefore, overproduction of IL-1 $\beta$ exacerbated shear stress-induced ER stress-mediated apoptosis via the IRAK2/CHOP signaling pathway in endothelial cells.
\end{abstract}

Correspondence to: Professor Gang Wang, Department of Emergency Medicine, The Second Affiliated Hospital of Xi'an Jiaotong University, 157 Xiwu Road, Xi'an, Shaanxi 710004, P.R. China

E-mail: jgowhv@sina.com

Key words: endothelial cells, endoplasmic reticulum stress, apoptosis, inflammation

\section{Introduction}

A number of pathogenic factors, including environmental risk factor exposure, aging, genetic defects and metabolic disorders, are frequently associated with the development of atherosclerosis. As atherosclerotic lesions primarily emerge and develop locally at typical arterial regions, including branched, bifurcated and curvature sites, the local hemodynamics of arteries maybe taken into consideration when investigating the pathogenesis of atherosclerosis (1). Generally, two types of blood flow patterns characterize the local arterial hemodynamics: The steady laminar blood flow (s-flow), and the disturbed blood flow (d-flow) (2). Vascular regions dominated by s-flow are relatively atheroresistant; however, d-flow, comprising transient flow, separated flow and reversal, frequently results in shear stress, which makes the subjected vascular region atherosusceptible (3).

Vascular endothelial cells (ECs) form the inner wall of arterial vessels, and are therefore directly exposed to the blood flow. Under mechanical stimuli, including d-flow, ECs modulate various biological responses, including vasoconstriction maladjustment, local inflammation, oxidative stress and cellular apoptosis (4). Injury to the arterial endothelium serves an important role in the development of inflammation, redox and coagulation, making the vessel atherosusceptible (5). A variety of signal transduction pathways may lead to the apoptosis of ECs. It has previously been demonstrated that the mitochondria-induced, death receptor-induced and endoplasmic reticulum stress-induced pathways are the three primary terminal apoptotic signaling pathways (6-8).

The majority of newly-synthesized proteins are folded and matured in the endoplasmic reticulum (ER). When the cell is exposed to stressful conditions, the folding and maturation processes cease and the unfolded protein response is initiated. When the accumulation of unfolded/misfolded proteins exceeds the capacity of the ER to correct the assembly of abnormally-folded proteins, the ER stress response is triggered to provoke multiple pathological alterations (9). As one of the molecular chaperones of ER, $78 \mathrm{kDa}$ glucose-regulated protein (GRP78) binds to stress sensors of the ER (serine/threonine 
protein kinase/endoribonuclease IRE1, eukaryotic translation initiation factor 2- $\alpha$ kinase 3 and cyclic AMP-dependent transcription factor ATF6- $\alpha$ ) to keep the apoptotic signaling inactive under normal physiological conditions. However, under stressful conditions, GRP78 disassociates from the stress signal transducers to trigger the activation of their signaling pathways. Previous studies have demonstrated that ER stress may induce cellular apoptosis through the DNA damage-inducible transcript 3 protein (CHOP) pathway (10). Activated CHOP is able to initiate the translocation of caspase 12 from the ER to the plasma in order to trigger the activation of the caspase cascade (11). CHOP is generally considered to be a pro-apoptotic factor (12). A previous study demonstrated that exposure of cultured ECs to d-flow results in an increase in the expression level of GRP78, indicating that d-flow is one of the pathological initiators of ER stress. These previous results formed the basis of the investigation into the involvement of ER stress in the apoptosis of ECs exposed to d-flow (13).

In addition, it has been hypothesized that inflammation may be important in accelerating and exacerbating the formation and rupture of atherosclerotic plaques (14). Inflammatory cytokines, including tumor necrosis factor- $\alpha$ (TNF- $\alpha$ ) and interleukins (ILs) are important transducers and amplifiers of these processes (15). It was reported that TNF- $\alpha$ may further recruit and stimulate the expression of other ILs to exacerbate inflammation (16). The IL-1 family is associated with the occurrence and development of numerous inflammatory diseases. A previous study demonstrated that the use of antagonists, including interleukin-1 receptor antagonist protein (IL-1Ra) and interleukin-36 receptor antagonist protein was able to relieve local and systemic inflammation (17). A recent study observed that IL-1 $\beta$ promoted ER stress-induced cellular apoptosis via interleukin-1 receptor associated kinase 2 (IRAK2) (18). In the present study, using an artificial in vitro model to mimic d-flow, cultured human aortic endothelial cells (HAECs) were exposed to shear stress. By using an ER stress inhibitor and specific small interfering (si)RNA against IRAK2, the involvement of IRAK2/CHOP signaling in ER stress-mediated shear stress-induced cellular apoptosis was investigated.

\section{Materials and methods}

Cell culture and treatment. HAECs were purchased from the Type Culture Collection of the Chinese Academy of Sciences (Shanghai, China) and cultured in Dulbecco's modified Eagle's medium (Gibco; Thermo Fisher Scientific, Inc., Waltham, MA, USA) supplemented with $10 \%$ fetal bovine serum (Gibco; Thermo Fisher Scientific, Inc.). L-glutamine (2 mmol/l; Invitrogen; Thermo Fisher Scientific, Inc.) and antibiotics (1\% penicillin/streptomycin; Invitrogen; Thermo Fisher Scientific, Inc.). Cells were cultured in a cell incubator providing a humidified environment, with $5 \% \mathrm{CO}_{2}$ and $95 \%$ air at $37^{\circ} \mathrm{C}$. Equal amounts of cells were divided into 7 groups, namely the control group (Control), 4-phenylbutyrate (4-PBA)-treated group (4-PBA), d-flow-exposed group (d-flow), siRNA-treated group (siRNA), IL-1Ra-treated d-flow-exposed group (IL-1Ra+d-flow), 4-PBA-treated d-flow-exposed group (4-PBA+d-flow) and siRNA treated d-flow-exposed group (d-flow+siRNA). Cell treatments are listed in Table I.
Target gene silencing using siRNA. In accordance with a previous study, IRAK2 in HAECs was silenced using siRNA (18). The sequence of the siRNA against IRAK2 was 5'-CTTCGCCTCCTACGTGATCAC-3', and was acquired from Shanghai GenePharma Co., Ltd. (Shanghai, China). A scramble sequence, 5'-GAACAGACGACGTTGACAA-3', was used as the scramble control. According to the manufacturer's protocol, using HiPerFect siRNA transfection reagent (Qiagen $\mathrm{GmbH}$, Hilden, Germany), the siRNAs were transfected into HAECs at final concentrations of $12.5 \mathrm{mmol} / \mathrm{l}$. Cells were used for subsequent experiments following $24 \mathrm{~h}$ culturing.

Artificial d-flow model. The artificial d-flow model was created using a cone-and-plate chamber according to the description in a previous report (19). This apparatus consists of a cone rotating about its center axis in a tissue culture dish to produce stable laminar flow. The components of the cone-and-late apparatus were produced at the College of Machinery of Xi'an Jiaotong University (Xi'an, China). The HAECs were exposed to d-flow for $0.5,1,2,3$ and $4 \mathrm{~h}$.

Cellular apoptosis assay. Cellular apoptosis was detected using a terminal deoxynucleotidyl transferase dUTP nick end labeling (TUNEL) assay. The In Situ Cell Death Detection kit (Roche Diagnostics, Basel, Switzerland) was used to perform the detection. Following fixation with $4 \%$ paraformaldehyde for $12 \mathrm{~h}$ at room temperature (Sigma-Aldrich; Merck KGaA, Darmstadt, Germany), cultured HAECs were permeabilized using 1\% Triton X-100 (Sigma-Aldrich; Merck KGaA). Cells were subsequently treated with TUNEL staining solution for $1 \mathrm{~h}$ at $37^{\circ} \mathrm{C}$ in accordance with manufacturer's protocol. After washing the slides five times in PBS and mounted with $3 \%$ hydrogen peroxide in formaldehyde, a fluorescence microscope was used to observe TUNEL positive cells. Five fields of view were selected.

IL-1 $\beta$ concentration detection. IL-1 $\beta$ concentration in the cell culture supernatants was measured using an ELISA assay, with a Quantkine Rat IL-1 $\beta$ ELISA kit (catalog no. DLB50; R\&D Systems, Inc., Minneapolis, MN, USA). The measurements were carried out according to the manufacturer's protocol. Standard curves and absorbance values were used to calculate the concentrations.

Western blotting. Harvested HAECs were washed and lysed in radioimmunoprecipitation assay buffer (Santa Cruz Biotechnology, Inc., Dallas, TX, USA) supplemented with phenylmethylsulfonyl fluoride $(0.5 \mathrm{mmol} / \mathrm{l}$; Santa CruzBiotechnology, Inc.), dithiothreitol (1 mmol/l; Beyotime Institute of Biotechnology, Haimen, China) and protease inhibitor $(150 \mathrm{mmol} / \mathrm{l}$; Invitrogen; Thermo Fisher Scientific, Inc.). Protein concentrations were determined using the colorimetric Bradford assay (Pierce; Thermo Fisher Scientific, Inc.). A total of $10 \mu$ g protein was loaded onto $10 \%$ gels and subjected to SDS-PAGE, and the loaded proteins were separated by vertical electrophoresis. Proteins were subsequently electronically transferred to polyvinylidene fluoride or nitrocellulose membranes (EMD Millipore, Billerica, MA, USA). Following blocking for $1 \mathrm{~h}$ in $5 \%$ non-fat milk in TBST for $1 \mathrm{~h}$ at $25^{\circ} \mathrm{C}$, specific antibodies against IL-1 $\beta$ (catalog no. sc-130323; Santa 
Table I. Cell grouping and treatments.

\begin{tabular}{lll}
\hline Groups & Treatment reagent & \multicolumn{1}{c}{ Description } \\
\hline Control & Medium & Treated with control medium \\
4-PBA & 4-PBA & Treated with 4-PBA at concentration of 0.6 mmol/1 \\
siRNA & siRNA & Specific siRNA against IRAK2 \\
d-flow & d-flow & Artificial d-flow model \\
d-flow+IL-1Ra & IL-1Ra and d-flow & Treated with IL-1Ra at concentration of 200 ng/ml \\
& & when exposed to d-flow \\
d-flow+4PBA & 4-PBA and d-flow & Treated with 4-PBA when exposed to d-flow \\
d-flow+siRNA & siRNA and d-flow & Pretreated with siRNA against IRAK2 and subse \\
& & quently exposed to d-flow
\end{tabular}

4-PBA, 4-phenylbutyric acid; d-flow, disturbed blood flow; siRNA, small interfering RNA; IL-1Ra, interleukin-1 receptor antagonist protein.

Cruz Biotechnology, Inc.; dilution 1:2,000), GRP78 (catalog no. 3183; 1:2,000; Cell Signaling Technology, Inc., Danvers, MA, USA), IRAK2 (catalog no. ab62419; 1:1,000; Abcam, Cambridge, UK), CHOP (catalog no. ab10444; 1:2,000; Abcam), caspase 12 (catalog no. ab62484; 1:1,000; Abcam) and GAPDH (catalog no. ab8245; 1:5,000; Abcam) were used to incubate the membranes for $12 \mathrm{~h}$ at $4^{\circ} \mathrm{C}$. Corresponding horseradish peroxidase-conjugated secondary antibodies (catalog no. ab7010, Abcam; catalog no. sc2364 and sc3747; Santa Cruz Biotechnology, Inc.) were used to incubate the membranes for $1 \mathrm{~h}$ at room temperature, which were illuminated using SuperSignal West Pico enhanced chemiluminescence reagents (Pierce; Thermo Fisher Scientific, Inc.).

Statistical analysis. Data acquired in the present study are presented as the mean \pm standard deviation of three independent experiments. The data were analyzed using SPSS software, version 16.0 (SPSS, Inc., Chicago, IL, USA). Differences between groups were analyzed using one-way analysis of variance followed by Bonferroni's post hoc test. $\mathrm{P}<0.05$ was considered to indicate a statistically significant difference.

\section{Results}

d-flow triggers the activation of ER stress, which induces $H A E C$ apoptosis in a time-dependent manner. As presented in Fig. 1, following exposure to d-flow, ER stress-mediated apoptotic signaling was observed to be activated in HAECs in a time-dependent manner. The expression levels of the molecular markers of this pathway, GRP78 and CHOP, were observed to be significantly increased in a time-dependent manner. The actual time-dependent d-flow induced apoptosis was observed using a TUNEL assay.

Exposure to d-flow increases the synthesis and secretion of $I L-1 \beta$ in cultured HAECs, in a time-dependent manner. As demonstrated in Fig. 2 , the synthesis of IL-1 $\beta$ was significantly increased in d-flow-treated HAECs in a time-dependent manner, which was evidenced by an increased protein expression level in harvested HAECs. Additionally, the secretion of IL-1 $\beta$ in d-flow-treated HAECs was observed to be increased

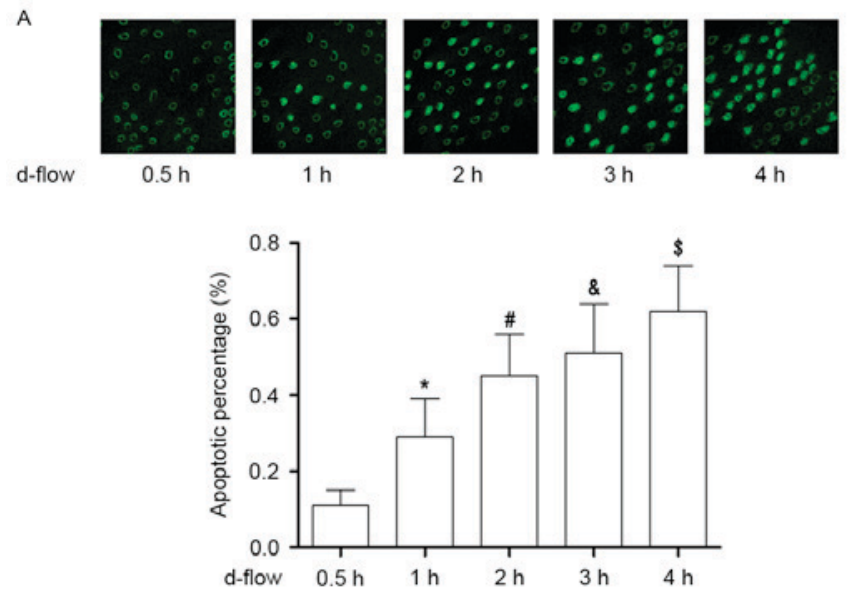

B
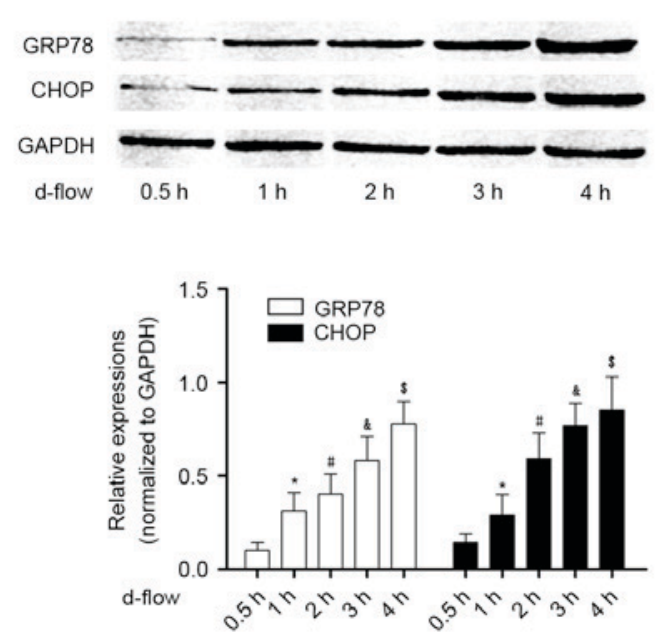

Figure 1. Analysis of apoptosis in HAECs exposed to d-flow. (A) The upper panel demonstrates the captured images from the terminal deoxynucleotidyl transferase dUTP nick end labeling assay of HAECs treated with d-flow for $0.5,1,2,3$ and $4 \mathrm{~h}$. The graph on the lower panel indicates the detected apoptotic percentage of HAECs. Magnification, x200. (B) The upper panel exhibits the immunoblotting analysis of GRP78, CHOP and GAPDH in HAECs treated with d-flow for $0.5,1,2,3$ and $4 \mathrm{~h}$. The graph on the lower panel presents the relative expression levels of GRP78 (white columns) and CHOP (black columns), with GAPDH as the internal reference. ${ }^{*} \mathrm{P}<0.05$ vs. $0.5 \mathrm{~h} ;{ }^{\#} \mathrm{P}<0.05$ vs. $1 \mathrm{~h} ;{ }^{\circledR} \mathrm{P}<0.05$ vs. $2 \mathrm{~h} ;{ }^{\$} \mathrm{P}<0.05$ vs. $3 \mathrm{~h}$. HAECs, human aortic endothelial cells; d-flow, disturbed blood flow; GRP78, $78 \mathrm{kDa}$ glucose-regulated protein; CHOP, DNA damage-inducible transcript 3 protein; d-flow, disturbed blood flow. 
A
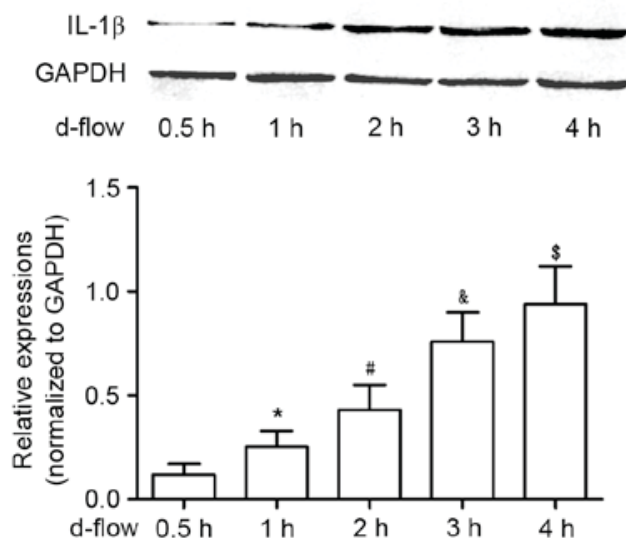

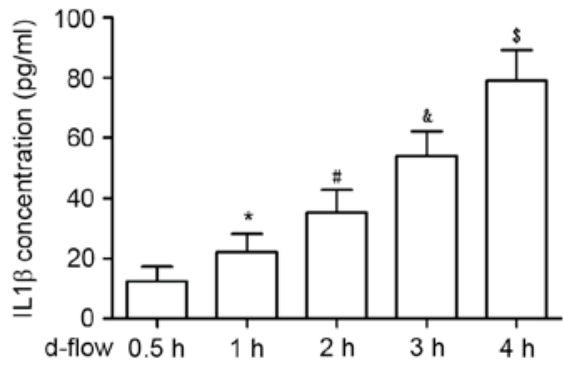

Figure 2. Analysis of IL-1 $\beta$ expression in HAECs exposed to d-flow. (A) The upper panel exhibits the representative image of the western blotting of IL-1 $\beta$ and GAPDH in HAECs treated with d-flow for $0.5,1,2,3$ and $4 \mathrm{~h}$. The graph on the lower panel indicates the relative expression of IL-1 $\beta$ in HAECs, with GAPDH as the internal reference. (B) Results of the ELISA assay of IL-1 $\beta$. The graph exhibits the detected IL-1 $\beta$ concentrations in the supernatant of cultured HAECs treated with d-flow for $0.5,1,2,3$ and $4 \mathrm{~h} .{ }^{*} \mathrm{P}<0.05$ vs. $0.5 \mathrm{~h} ;{ }^{\#} \mathrm{P}<0.05$ vs. $1 \mathrm{~h} ;{ }^{\&} \mathrm{P}<0.05 \mathrm{vs} .2 \mathrm{~h} ;{ }^{~} \mathrm{P}<0.05$ vs. $3 \mathrm{~h}$. HAECs, human aortic endothelial cells; IL-1 $\beta$, interleukin-1 $\beta$; d-flow, disturbed blood flow.

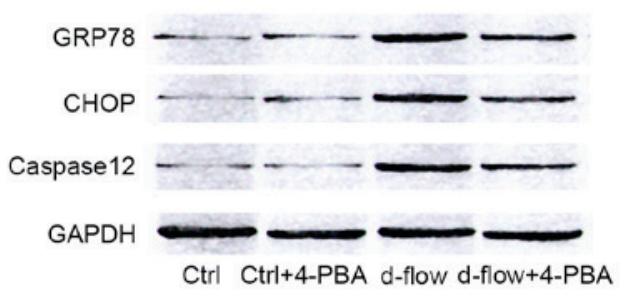

B

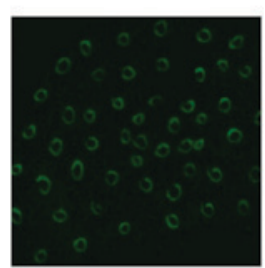

Ctrl

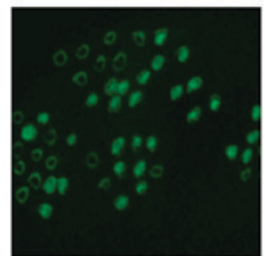

d-flow

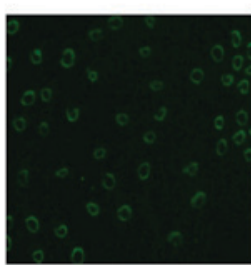

Ctrl+4-PBA

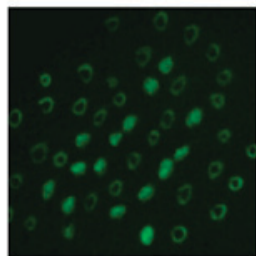

d-flow+4-PBA
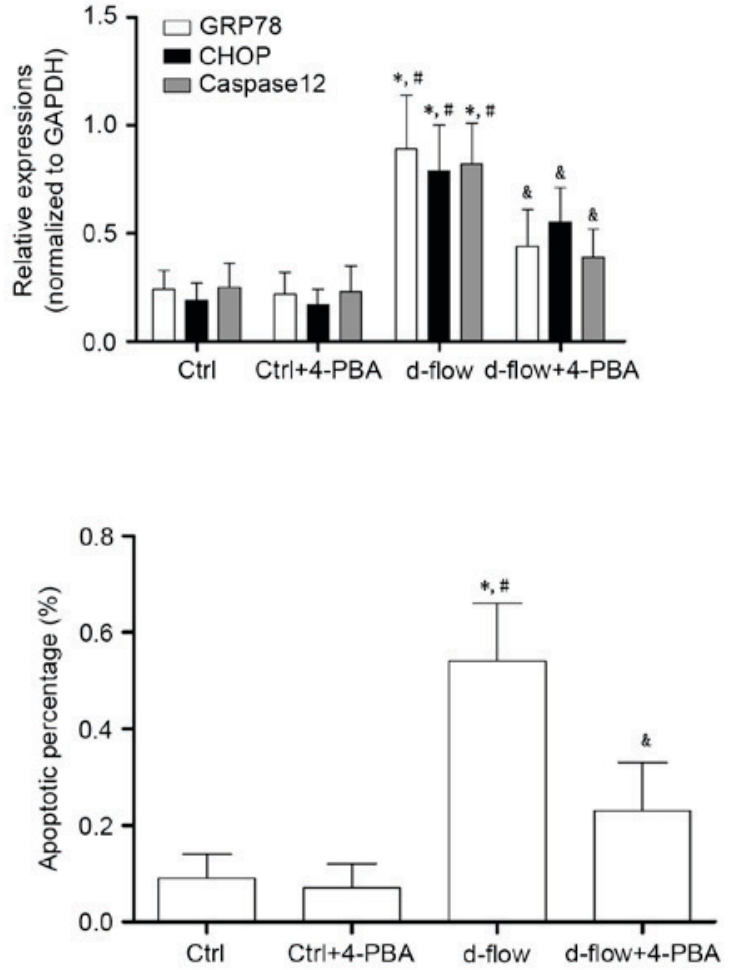

Figure 3. Analysis of the effects of treatment with 4-PBA in HAECs exposed to d-flow. (A) Results of western blotting of GRP78, CHOP, caspase 12 and GAPDH in HAECs treated with 4-PBA and/or d-flow. The relative expression of GRP78 (white columns) and CHOP (black columns) in HAECs treated with 4-PBA and/or d-flow was normalized to GAPDH. (B) The left panel exhibits the captured images from the terminal deoxynucleotidyl transferase dUTP nick end labeling assay of HAECs treated with 4-PBA and/or d-flow. The detected apoptotic percentages of HAECs treated with 4-PBA and/or d-flow were quantified. Magnification, x200. " $\mathrm{P}<0.05$ vs. Ctrl; ${ }^{\mathrm{P}}<0.05$ vs. Ctrl+4-PBA; ${ }^{\circledR} \mathrm{P}<0.05$ vs. d-flow. HAECs, human aortic endothelial cells; d-flow, disturbed blood flow; GRP78, 78 kDa glucose-regulated protein; CHOP, DNA damage-inducible transcript 3 protein; 4-PBA, 4-phenylbutyric acid; Ctrl, control.

in a time-dependent manner, evidenced by increased protein expression in the supernatant of cultured HAECs.

4-PBA incubation attenuates d-flow-mediated HAEC death by suppressing ER stress. Treatment with 4-PBA treatment significantly decreased the expression of GRP78 and CHOP, which were considered to be molecular markers of ER stress-mediated apoptotic signaling. Additionally, results from the TUNEL assay and the expression levels of caspase 12 demonstrated that the d-flow induced ER stress mediated apoptosis was inhibited by 4-PBA in HAECs (Fig. 3). 


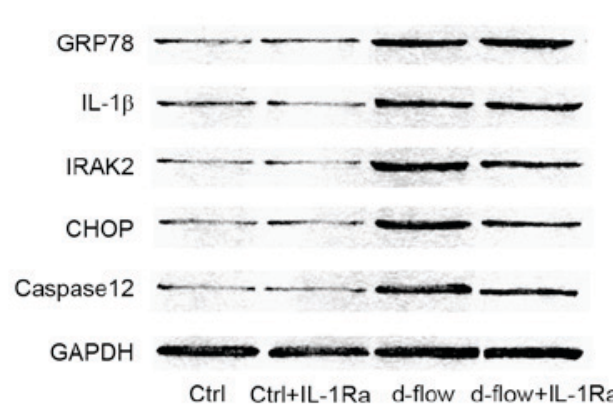

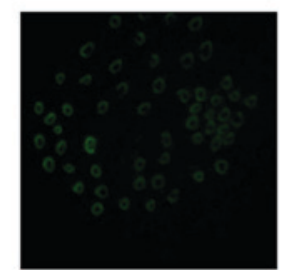

Ctrl

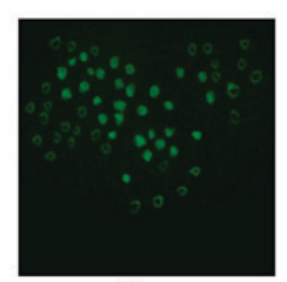

d-flow

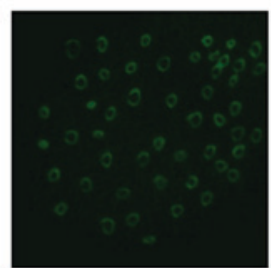

Ctrl+IL-1Ra

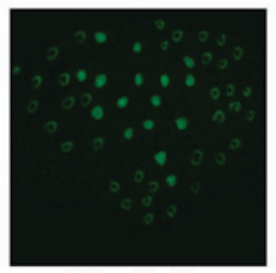

d-flow+IL-1Ra
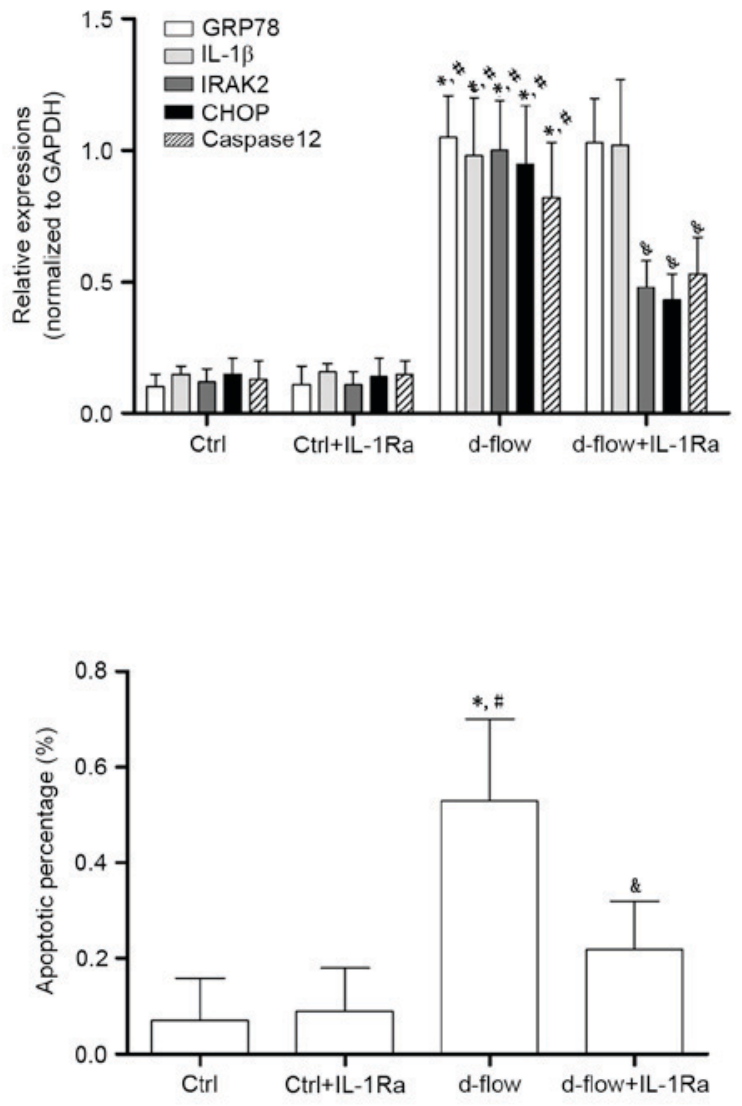

Figure 4. Analysis of the effects of treatment with IL-1Ra in HAECs exposed to d-flow. (A) Results of the western blotting of GRP78, IL-1 $\beta$, IRAK2, CHOP, caspase 12 and GAPDH in HAECs treated with IL-1Ra and/or d-flow. The relative expressions of GRP78 (white columns), IL-1 $\beta$ (light gray columns), IRAK2 (dark gray columns) and CHOP (black columns) were normalized to GAPDH. (B) Images were captured of the terminal deoxynucleotidyl transferase dUTP nick end labeling assay of HAECs treated with IL-1Ra and/or d-flow. The detected apoptotic percentages of HAECs treated by IL-1Ra and/or d-flow were quantified. Magnification, x200. "P<0.05 vs. Ctrl; ${ }^{\#} \mathrm{P}<0.05$ vs. Ctrl+IL-1Ra; ${ }^{\circledR} \mathrm{P}<0.05$ vs. d-flow. IL-1Ra, interleukin-1 receptor antagonist protein; GRP78, 78 kDa glucose-regulated protein; CHOP, DNA damage-inducible transcript 3 protein; d-flow, disturbed blood flow; HAECs, human aortic endothelial cells; Ctrl, control; IRAK2, interleukin-1 receptor associated kinase 2; IL-1 $\beta$, interleukin-1 $\beta$.

IL-1Ra incubation lowers the apoptotic rate of d-flow-exposed HAECs by inhibiting IRAK2/CHOP signaling. The association between IL-1Ra and alterations in d-flow-induced ER stress-mediated signal transduction and actual apoptosis are demonstrated in Fig. 4. IL-1Ra did not affect cellular apoptosis (determined by TUNEL assay and caspase12 expression level) in untreated HAECs. However, the d-flow-induced apoptosis was significantly inhibited by IL-1Ra administration. It was additionally observed that in d-flow-treated HAECs, IL-1Ra treatment decreased the expression of IRAK2 and CHOP without affecting the expression levels of GRP78 and IL-1 $\beta$. The results of the present study indicated that the IL-1Ra inhibited apoptotic signal transduction during ER stress by affecting the signal transduction of IRAK2/CHOP.

siRNA against IRAK2 attenuates $d$-flow-mediated HAEC apoptosis by blocking IRAK2/CHOP signaling. In order to further confirm the role of IRAK2/CHOP signaling in mediating d-flow-induced HAEC apoptosis, RNA interference was performed. siRNA against IRAK2 was transfected into HAECs. As presented in Fig. 5, IRAK2 siRNA transfection exhibited apparent inhibitory effects on cellular apoptosis (determined by TUNEL assay and caspase 12 expression level analysis) induced by d-flow. IRAK2 siRNA transfection markedly inhibited the expression levels of $\mathrm{CHOP}$ in d-flow treated HAECs. However, the expression levels of GRP78 and IL-1 $\beta$ were not affected by IRAK2 siRNA transfection.

\section{Discussion}

Atherosclerotic vascular disease-associated mortality represents a large proportion of cerebrovascular and cardiovascular mortalities worldwide. In the arterial tree, distinct lesions may be observed in different regions due to regional differences in shear stress forces produced by blood flow (20). A previous study indicated that exposure to d-flow triggers pathological alterations which render ECs dysfunctional and proatherogenic (21). Apoptosis in the endothelium modulates biological alterations within the vessel wall, including deregulation of vascular wall remodeling, decreased production of vasorelaxant substances and initiation of the coagulation cascade and pro-inflammatory processes (22-24). These alterations have been hypothesized to be the mechanisms underlying atherosclerosis. In the present study, cultured HAECs were exposed to d-flow generated by an artificial device. It was observed that 


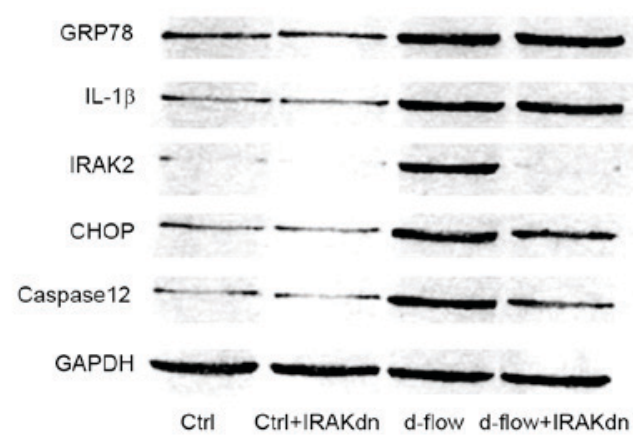

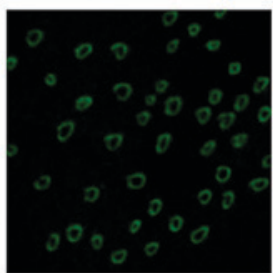

Ctrl

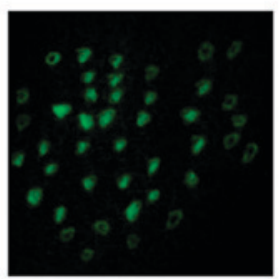

d-flow

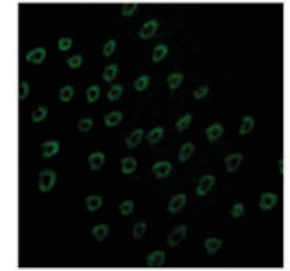

Ctrl+IRAKdn

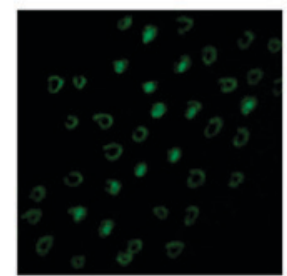

d-flow+IRAKdn
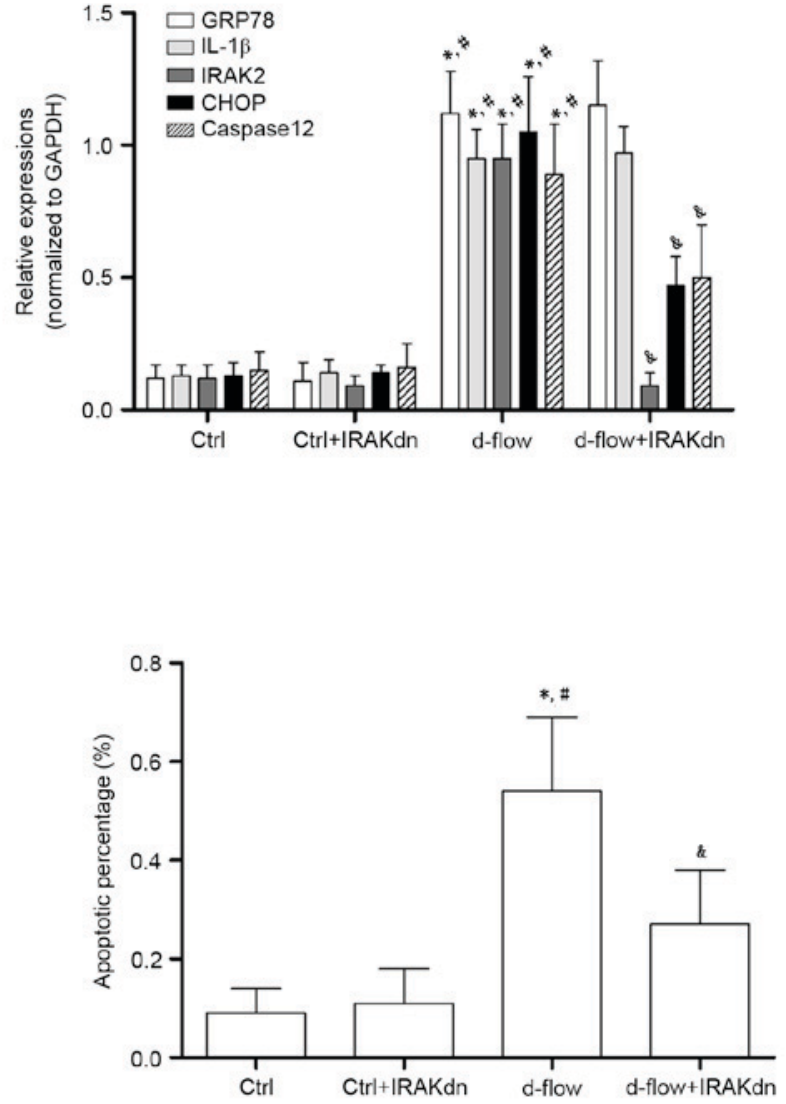

Figure 5. Analysis of the effects of treatment with IRAK2 siRNA in HAECs exposed to d-flow. (A) Results of the immunoblotting of GRP78, IL-1 $\beta$, IRAK2, CHOP, caspase 12 and GAPDH in HAECs treated with IRAK2 siRNA and/or d-flow. The relative expression of GRP78 (white columns), IL-1 $\beta$ (light gray columns), IRAK2 (dark gray columns) and CHOP (black columns) was normalized to GAPDH. (B) Images were captured of the terminal deoxynucleotidyl transferase dUTP nick end labeling assay of HAECs treated with IRAK2 siRNA and/or d-flow. The detected apoptotic percentages of HAECs treated with IRAK2 siRNA and/or d-flow were quantified. Magnification, x200. * $\mathrm{P}<0.05$ vs. Ctrl; ${ }^{\text {P }}<0.05$ vs. Ctrl+IRAKdn; ${ }^{\circledR} \mathrm{P}<0.05$ vs. d-flow. GRP78, $78 \mathrm{kDa}$ glucose-regulated protein; CHOP, DNA damage-inducible transcript 3 protein; d-flow, disturbed blood flow; HAECs, human aortic endothelial cells; Ctrl, control; IRAK2, interleukin-1 receptor associated kinase 2; IL-1 $\beta$, interleukin-1 $\beta$; IRAKdn, IRAK downregulated; siRNA, small interfering RNA.

d-flow induced the apoptosis of HAECs in a time-dependent manner.

The ER is an important cellular organelle, primarily involved in directing protein folding, modification and maturation. Under certain pathological circumstances, when ER stress is severe and prolonged, signal transductions are activated to trigger multiple biological effects (25). In vivo and in vitro investigations have demonstrated the association between ER stress and atherosclerosis (26). Administration of an exogenous ER stress activator was observed to promote the formation of atherosclerotic plaques (27). Evidence of ER stress activation has additionally been observed within the human atherosclerotic endothelium (28). In addition, ER stress was demonstrated to induce apoptosis in endothelial cells (29). Therefore, it may be hypothesized that d-flow-induced EC apoptosis is mediated by ER stress. The present study demonstrated that the expression of GRP78, considered to be the chaperone of ER stress, was increased significantly in HAECs following exposure to d-flow. The expression of the pro-apoptotic factor of ER stress, CHOP, was additionally observed to be elevated in d-flow-treated HAECs. The results of the present study indicated that d-flow-induced apoptosis was mediated by ER stress. 4-PBA is one of the specific suppressors of ER stress. In the present study, treatment with 4-PBA inhibited the increased expression levels of GRP78 and CHOP. Therefore, 4-PBA impaired d-flow-induced ER stress-mediated HAEC apoptosis. The results of the present study further supported the hypothesis that d-flow-induced HAEC apoptosis is mediated by ER stress.

It is widely accepted that atherosclerosis is induced and promoted by molecular and cellular pathways of inflammation. Numerous inflammatory cytokines, including TNF- $\alpha$, tumor growth factor- $\beta$ and IL-1 provide mechanisms that may be able to alter the arterial biology of the endothelium to favor plaque formation and atherothrombotic events (30). In a previous study, inflammation and ER stress were hypothesized to be associated as they share a number of effectors and regulators (31). A 'vicious cycle' was proposed, since inflammatory and ER stress signals were observed to exacerbate cellular apoptosis via their interactions (32). In the present study, it was demonstrated that, following d-flow exposure, expression level of IL-1 $\beta$ in HAECs and their supernatant increased 
significantly. ER stress and inflammatory signaling were simultaneously initiated. However, following treatment with the IL-1 $\beta$ receptor antagonist IL-1Ra, cellular apoptosis was attenuated. IL-1Ra treatment decreased the expression level of CHOP in d-flow-treated cells. The results of the present study indicated that IL-1 $\beta$ promoted ER stress-mediated cellular apoptosis via the CHOP signaling pathway.

By binding to its receptor IL-1R, IL-1 $\beta$ activates downstream signaling transduction by forming the IL-1R-AcP complex with an accessory protein (AcP). The C-terminal death domain of this complex couples IRAKs and subsequently activates TNF receptor associated factor- 6 to initiate the apoptotic pathway $(33,34)$. The close association between ER stress-mediated apoptosis and IRAK2 was identified by a previous study (18), the results of which demonstrated that the expression of IRAK2 increases in conditions of ER stress. In addition, increased IRAK2 promotes CHOP expression, which transduces the death signal in ER stress-mediated apoptosis. In the present study, RNA interference was used to knockdown the expression of IRAK2. The results of the present study demonstrated that the knockdown of IRAK2 significantly inhibited the d-flow-induced apoptosis of HAECs. It was additionally observed that the knockdown of IRAK 2 suppressed the expression of CHOP without an apparent affect on GRP78 and IL-1 $\beta$. The results of the present study indicated that the secreted IL- $1 \beta$ in d-flow-treated HAECs was involved in exacerbating apoptosis through the IRAK2/CHOP signaling pathway.

In conclusion, by activating ER stress, exposure to d-flow induced HAEC apoptosis via the CHOP signaling pathway. The inflammatory cytokine IL-1 $\beta$ was produced in d-flow-treated HAECs. IL-1 $\beta$ exacerbated d-flow-induced cellular apoptosis. In addition, the apoptotic signal was transduced via the IL-1 $\beta /$ IRAK2/CHOP signaling pathway. This signaling pathway may be considered to be crosstalk between ER stress and inflammation under conditions of disturbed blood flow. The results of the present study may provide a theoretical basis for the identification of potential molecular targets for the treatment of atherosclerosis.

\section{Acknowledgements}

The present study was supported by the project fund of Shaanxi Province Science and Technology Key Projects (grant no. 2016YFJH2-05).

\section{References}

1. Gimbrone MA Jr and García-Cardeña G: Vascular endothelium, hemodynamics, and the pathobiology of atherosclerosis. Cardiovasc Pathol 22: 9-15, 2013.

2. Heo KS, Le NT, Cushman HJ, Giancursio CJ, Chang E, Woo CH, Sullivan MA, Taunton J, Yeh ET, Fujiwara K and Abe J: Disturbed flow-activated p90RSK kinase accelerates atherosclerosis by inhibiting SENP2 function. J Clin Invest 125: 1299-1310, 2015.

3. Xu Q: Disturbed flow-enhanced endothelial turnover in atherosclerosis. Trends Cardiovasc Med 19: 191-195, 2009.

4. Heo KS, Fujiwara K and Abe J: Disturbed-flow-mediated vascular reactive oxygen species induce endothelial dysfunction. Circ J 75: 2722-2730, 2011.

5. Van der Heiden K, Hierck BP, Krams R, de Crom R, Cheng C, Baiker M, Pourquie MJ, Alkemade FE, DeRuiter MC, Gittenberger-de Groot AC and Poelmann RE: Endothelial primary cilia in areas of disturbed flow are at the base of atherosclerosis. Atherosclerosis 196: 542-550, 2008.
6. Hida A, Kawakami A, Miyashita T, Yamasaki S, Nakashima K, Tanaka F, Izumi Y, Tamai M, Huang M, Ida $\mathrm{H}$, et al: Nitric oxide acts on the mitochondria and protects human endothelial cells from apoptosis. J Lab Clin Med 144: 148-155, 2004.

7. Qiu ZL, Zhang JP and Guo XC: Endoplasmic reticulum stress and vascular endothelial cell apoptosis. Zhongguo Yi Xue Ke Xue Yuan Xue Bao 36: 102-107, 2014 (In Chinese).

8. Skurk C and Walsh K: Death receptor induced apoptosis: A new mechanism of homocysteine-mediated endothelial cell cytotoxicity. Hypertension 43: 1168-1170, 2004.

9. Pluquet O, Pourtier A and Abbadie C: The unfolded protein response and cellular senescence. A review in the theme: Cellular mechanisms of endoplasmic reticulum stress signaling in health and disease. Am J Physiol Cell Physiol 308: C415-C425, 2015.

10. Ma J, Qiu Y, Yang L, Peng L, Xia Z, Hou LN, Fang C, Qi H and Chen HZ: Desipramine induces apoptosis in rat glioma cells via endoplasmic reticulum stress-dependent CHOP pathway. J Neurooncol 101: 41-48, 2011.

11. Lakshmanan AP, Thandavarayan RA, Palaniyandi SS, Sari FR, Meilei H, Giridharan VV, Soetikno V, Suzuki K, Kodama M and Watanabe K: Modulation of AT-1R/CHOP-JNK-Caspase12 pathway by olmesartan treatment attenuates ER stress-induced renal apoptosis in streptozotocin-induced diabetic mice. Eur J Pharm Sci 44: 627-634, 2011.

12. Nakagawa T, Zhu H, Morishima N, Li E, Xu J, Yankner BA and Yuan J: Caspase-12 mediates endoplasmic-reticulum-specific apoptosis and cytotoxicity by amyloid-beta. Nature 403: 98-103, 2000.

13. Davies PF and Civelek M: Endoplasmic reticulum stress, redox, and a proinflammatory environment in athero-susceptible endothelium in vivo at sites of complex hemodynamic shear stress. Antioxid Redox Signal 15: 1427-1432, 2011.

14. Bäck M, Weber C and Lutgens E: Regulation of atherosclerotic plaque inflammation. J Intern Med 278: 462-482, 2015.

15. Dewberry R, Holden H, Crossman D and Francis S: Interleukin-1 receptor antagonist expression in human endothelial cells and atherosclerosis. Arterioscler Thromb Vasc Biol 20: 2394-2400, 2000.

16. Balato A, Di Caprio R, Canta L, Mattii M, Lembo S, Raimondo A, Schiattarella M, Balato N and Ayala F: IL-33 is regulated by TNF- $\alpha$ in normal and psoriatic skin. Arch Dermatol Res 306: 299-304, 2014

17. Mattii M, Ayala F, Balato N, Filotico R, Lembo S, Schiattarella M, Patruno C, Marone G and Balato A: The balance between pro- and anti-inflammatory cytokines is crucial in human allergic contact dermatitis pathogenesis: The role of IL-1 family members. Exp Dermatol 22: 813-819, 2013.

18. Liu Z, Zhao N, Zhu H, Zhu S, Pan S, Xu J, Zhang X, Zhang Y and Wang J: Circulating interleukin-1 $\beta$ promotes endoplasmic reticulum stress-induced myocytes apoptosis in diabetic cardiomyopathy via interleukin-1 receptor-associated kinase-2. Cardiovasc Diabetol 14: 125, 2015.

19. Reinhart-King CA, Fujiwara K and Berk BC: Physiologic stress-mediated signaling in the endothelium. Methods Enzymol 443: 25-44, 2008.

20. Heo KS, Fujiwara K and Abe J: Shear stress and atherosclerosis. Mol Cells 37: 435-440, 2014.

21. Davies PF, Civelek M, Fang Y, Guerraty MA and Passerini AG: Endothelial heterogeneity associated with regional athero-susceptibility and adaptation to disturbed blood flow in vivo. Semin Thromb Hemost 36: 265-275, 2010.

22. Zhang T, Tian F, Wang J, Jing J, Zhou SS and Chen YD: Atherosclerosis-associated endothelial cell apoptosis by MiR-429-mediated down regulation of Bcl-2. Cell Physiol Biochem 37: 1421-1430, 2015.

23. Zeng L, Zampetaki A, Margariti A, Pepe AE, Alam S, Martin D, Xiao Q, Wang W, Jin ZG, Cockerill G, et al: Sustained activation of XBP1 splicing leads to endothelial apoptosis and atherosclerosis development in response to disturbed flow. Proc Natl Acad Sci USA 106: 8326-8331, 2009.

24. Choy JC, Granville DJ, Hunt DW and McManus BM: Endothelial cell apoptosis: Biochemical characteristics and potential implications for atherosclerosis. J Mol Cell Cardiol 33: 1673-1690, 2001.

25. Cao SS and Kaufman RJ: Endoplasmic reticulum stress and oxidative stress in cell fate decision and human disease. Antioxid Redox Signal 21: 396-413, 2014. 
26. Hotamisligil GS: Endoplasmic reticulum stress and atherosclerosis. Nat Med 16: 396-399, 2010.

27. Tabas I: The role of endoplasmic reticulum stress in the progression of atherosclerosis. Circ Res 107: 839-850, 2010.

28. Ivanova EA and Orekhov AN: The role of endoplasmic reticulum stress and unfolded protein response in atherosclerosis. Int J Mol Sci 17: pii:E193, 2016.

29. Lu JP, Li X, Jin YL and Chen MX: Endoplasmic reticulum stress-mediated aldosterone-induced apoptosis in vascular endothelial cells. J Huazhong Univ Sci Technolog Med Sci 34: 821-824, 2014

30. Krams R, Segers D, Mousavi Gourabi B, Maat W, Cheng C, van Pelt C, van Damme LC, de Feyter P, van der Steen T, de Korte CL and Serruys PW: Inflammation and atherosclerosis: Mechanisms underlying vulnerable plaque. J Interv Cardiol 16: 107-113, 2003.
31. Dong Z, Zhou J, Zhang Y, Chen Y, Yang Z, Huang G, Chen Y, Yuan Z, Peng Y and Cao T: Astragaloside-IV alleviates Heat-induced inflammation by inhibiting endoplasmic reticulum stress and autophagy. Cell Physiol Biochem 42: 824-837, 2017.

32. Benosman S, Ravanan P, Correa RG, Hou YC, Yu M, Gulen MF, Li X, Thomas J, Cuddy M, Matsuzawa Y, et al: Interleukin-1 receptor-associated kinase-2 (IRAK2) is a critical mediator of endoplasmic reticulum (ER) stress signaling. PLoS One 8: e64256, 2013.

33. Cenni V, Sirri A, De Pol A, Maraldi NM and Marmiroli S: Interleukin-1-receptor-associated kinase 2 (IRAK2)-mediated interleukin-1-dependent nuclear factor kappaB transactivation in Saos2 cells requires the Akt/protein kinase B kinase. Biochem J 376: 303-311, 2003.

34. Meng Q, Zheng M, Liu H, Song C, Zhang W, Yan J, Qin L and Liu X: TRAF6 regulates proliferation, apoptosis, and invasion of osteosarcoma cell. Mol Cell Biochem 371: 177-186, 2012. 